Cholesterol Protects the Oxidized Lipid Bilayer from Water Injury : An All-Atom Molecular Dynamics Study

Owen, Michael C.

2018-06

Owen , M C , Kulig , W , Rog , T , Vattulainen , I \& Strodel , B 2018 , ' Cholesterol Protects the Oxidized Lipid Bilayer from Water Injury : An All-Atom Molecular Dynamics Study ' , Journal of Membrane Biology , vol. 251 , no. 3 , pp. 521-534 . https://doi.org/10.1007/s00232-018-0028-9

http://hdl.handle.net/10138/307513

https://doi.org/10.1007/s00232-018-0028-9

acceptedVersion

Downloaded from Helda, University of Helsinki institutional repository.

This is an electronic reprint of the original article.

This reprint may differ from the original in pagination and typographic detail.

Please cite the original version. 


\title{
Cholesterol Protects the Oxidized Lipid Bilayer From Water Injury: An All- Atom Molecular Dynamics Study
}

\author{
Michael C. Owen, ${ }^{[1]}$ Waldemar Kulig, ${ }^{[2]}$ Tomasz Rog, ${ }^{[2]}$ Ilpo Vattulainen, ${ }^{[2-4]}$ Birgit Strodel ${ }^{[1,5]}$ \\ ${ }^{[1]}$ Institute of Complex Systems: Structural Biochemistry (ICS-6), Forschungszentrum Jülich, 52425 Jülich, Germany. \\ ${ }^{[2]}$ Department of Physics, University of Helsinki, P.O. Box 64, FI-00014 Helsinki, Finland. \\ ${ }^{[3]}$ Department of Physics, Tampere University of Technology, P.O. Box 692, FI-33101 Tampere, Finland. \\ ${ }^{[4]}$ MEMPHYS - Center for Biomembrane Physics, University of Southern Denmark, Odense, Denmark. \\ ${ }^{[5]}$ Institute of Theoretical and Computational Chemistry, Heinrich Heine University Düsseldorf, Universitätsstrasse 1, \\ 40225 Düsseldorf, Germany.
}

\begin{abstract}
In an effort to delineate how cholesterol protects the membrane structure under oxidative stress conditions, we monitored the changes to the structure of lipid bilayers comprised of $30 \mathrm{~mol} \%$ cholesterol and an increasing concentration of Class B oxidized 1-palmitoyl-2oleoylphosphatidylcholine (POPC) glycerophospholipids, namely 1-palmitoyl-2-(9'-oxo-nonanoyl)sn-glycero-3-phosphocholine (PoxnoPC), and 1-palmitoyl-2-azelaoyl-sn-glycero-3-phosphocholine (PazePC), using atomistic molecular dynamics simulations. Increasing the content of oxidized phospholipids (oxPLs) from 0 to $60 \mathrm{~mol} \%$ oxPL resulted in the characteristic reduction in bilayer thickness and increase in area per lipid, thereby increasing the exposure of the membrane hydrophobic region to water. However cholesterol was observed to help reduce water injury by moving into the bilayer core and forming more hydrogen bonds with the oxPLs. Cholesterol also resists altering its tilt angle, helping to maintain membrane integrity. Water that enters the 1-nm-thick core region remains part of the bulk water on either side of the bilayer, with relatively few water molecules able to traverse through the bilayer. In cholesterol-rich membranes, the bilayer does not form pores at concentrations of $60 \mathrm{~mol} \%$ oxPL as was shown in previous simulations in the absence of cholesterol.
\end{abstract}

KEYWORDS: Lipid oxidation, cholesterol protection, oxidative stress, oxidized membranes, pore formation 


\section{INTRODUCTION}

The plasma membrane separates and regulates transport between the interior of cells and the outside environment, and thus controls internal conditions necessary to sustain life. Injuries to the membrane, whether at the atomic, macromolecular or cellular levels, can affect the form, function and survival of the cell to the detriment of the organism as a whole, whether single or multicellular. Oxidative stress is one source of injury which occurs when the cholesterol and phospholipid molecules that comprise the plasma membrane are oxidized by free radicals, such as $\mathrm{OH}^{\bullet}$ and $\mathrm{HO}_{2}$. These radicals can arise from incomplete combustion, as products of Fenton-type reactions, and can also be leaked from mitochondria. Moreover, following a heart attack, the myocardium can produce superoxide and hydrogen peroxide following its re-exposure to oxygen, and these radicals can form the hydroxyl radical in the presence of metal ions such as $\mathrm{Fe}^{2+}$ or $\mathrm{Cu}^{+}{ }^{1}$ In neuronal tissue, the hydroxyl radical can initiate the peroxidation of polyunsaturated fatty acids resulting in the production of activated aldehydes such as 4-hydroxy-2-nonenal, which can subsequently cause extensive cellular damage. $^{2}$

Given the important role of the membrane to cellular viability, it is no surprise that damage to the plasma membrane is associated with a wide range of diseases. Oxidative stress has been closely linked with cardiovascular, neurodegenerative and pulmonary diseases. ${ }^{3}$ Specifically, lipid oxidation has been shown to take place in cardiac atherosclerosis, ${ }^{4}$ and ischemia-reperfusion injury following heart attacks. ${ }^{1}$ Moreover, oxidative damage to lipids that surround a membrane protein, such as an ion transporter, can indirectly alter the function of membrane proteins and thus affect cellular regulation. ${ }^{5}$ A better understanding of the effects of oxidative stress on the structure of the plasma membrane could improve our understanding of these related diseases.

The plasma membrane is comprised mostly of phospholipids and cholesterol, both of which are amphiphilic. Glycerophospholipids are the most common phospholipids, and are comprised of sn-glycerol-3-phosphate, esterified at the $\mathrm{C} 1$ and $\mathrm{C} 2$ positions to chains and at the phosphate group 

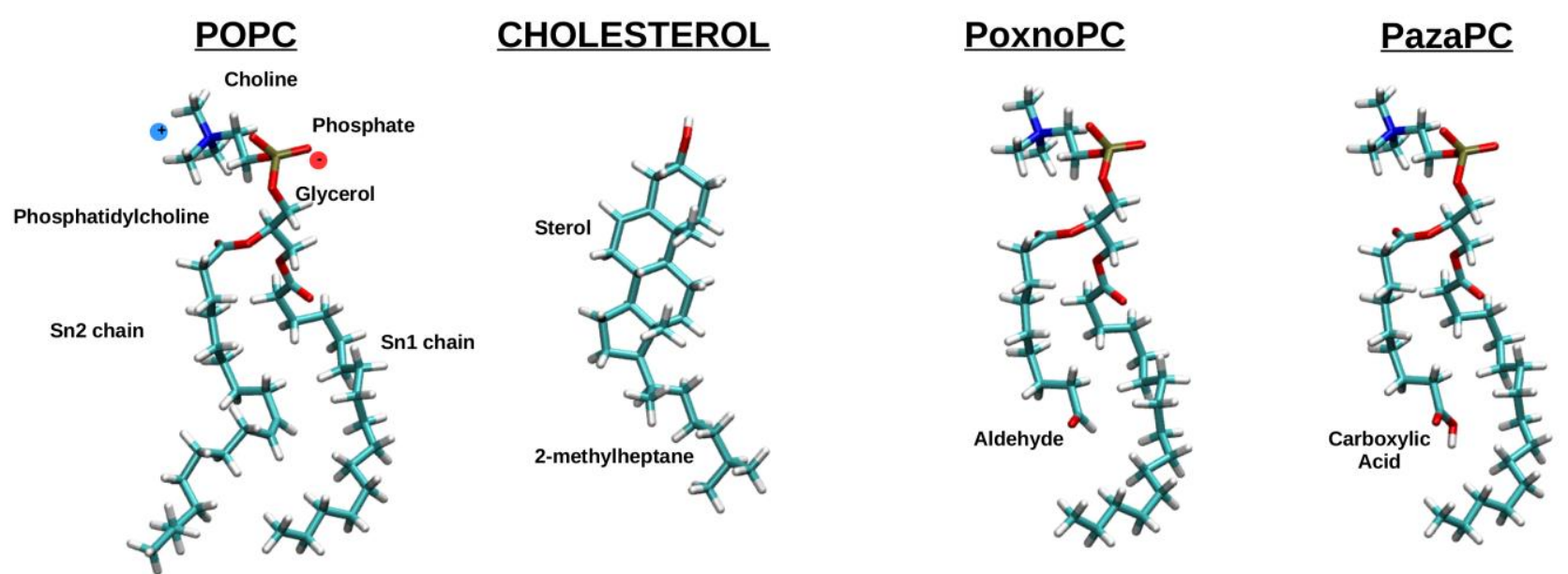

Figure 1. The four lipids used in this study: POPC (1-palmitoyl-2-oleoylphosphatidylcholine), cholesterol, PoxnoPC (1-palmitoyl-2-(9'-oxo-nonanoyl)-sn-glycero-3-phosphocholine), PazaPC (1-palmitoyl-2-azelaoylsn-glycero-3-phosphocholine). The choline, phosphate and glycerol, which together form the phosphatidylcholine are common to POPC, PoxnoPC, and PazaPC, whereas the latter two differ by the presence of an aldehyde or carboxylic acid group at the terminus of the sn-2 acyl chain. The sterol backbone and 2-methylheptane group of cholesterol are also shown.

to a hydrophilic group, most often choline ${ }^{6}$ The structure is described by stereospecific numbering (sn), which refers to the $\mathrm{C} 1$ and $\mathrm{C} 2$ of glycerol as the sn- 1 and sn- 2 position, respectively. Cholesterol is a derivative of cyclopentanoperhydrophenanthrene, the parent compound of steroids, with an additional hydroxyl group in ring $\mathrm{A}$, a double bond in ring $\mathrm{B}$, and a 2-methylheptane on ring $\mathrm{D}$ of the steroid backbone. The structures of both lipids are shown in Figure 1. Lipid oxidation can affect cholesterol, and the polar head groups and the unsaturated lipid tails of glycerophospholipids, with each having a different effect on the overall structure of the bilayer. ${ }^{7}$ Oxidation of the acyl chains yields oxidized phospholipids (oxPLs), which have been previously separated into two classes: Class A - hydroxy- or hydroxyperoxy-dieonyls, which contain an additional hydroxyl (-OH) or hydroxyperoxyl (-OOH) group, or Class B - those containing truncated acyl chains. ${ }^{8}$

Class B oxPLs such as those containing an aldehyde or carboxylic acid at their respective truncated sn-2 termini, have been shown to destabilize the bilayer structure in ways that can be easily related to disease pathology. Lipid asymmetry, where the lipid composition of one side of the bilayer 
is distinct from that of the other, plays an important role in the maintenance of the cell cycle, the regulation of apoptosis and also platelet coagulation. ${ }^{9}$ Class B oxPLs can induce lipid flip-flop, which affects the distribution of the lipids between the leaflets. ${ }^{10}$ Bilayers comprised of Class B oxPLs are more prone to disintegrate and aggregate into micelles than the corresponding Class A oxPLs with acyl chains of similar length. ${ }^{11}$ Class B oxPLs can also undergo lipid gymnastics and extend their acyl termini to the solvent phase in the extended conformation, though this only occurs with the carboxyl terminus. ${ }^{12,13}$ The extended conformation can facilitate interactions between the acyl chains and cytochrome c, which may affect the mechanism by which oxidative stress promotes apoptosis. ${ }^{14,15}$ Class B oxPLs induces more disruptive changes, as they can cause a larger decrease in bilayer thickness and deuterium order parameter than Class A oxPLs do. ${ }^{14,16}$ Furthermore, bilayers entirely comprised of Class B oxPLs easily become porous when all of oxPLs contain one truncated sn-2 acyl chain, and disintegrate completely when both acyl chains are truncated. ${ }^{17}$ Such extensive oxidation would cause the cell lose its capacity to regulate its contents and maintain its structure, which would ultimately lead to death by apoptosis or lysis. Cholesterol has been shown to attenuate these effects, but apart from its ability to co-localize with, and to slow down the lateral diffusion rates of oxPLs, little is known about the specific interactions cholesterol makes with other lipids as the oxidative load on the membrane increases. ${ }^{18,19}$

The amphiphilicity of glycerophospholipids arises from the polarity of the head group and the hydrophobicity of its acyl chains. In most mammalian cells, oleic acid, a chain of 18 carbon atoms with a single double bond is the most common fatty acid esterified at the sn- 2 position, ${ }^{20}$ whereas the fully saturated, 16-carbon, palmitic acid is most frequently found at the sn-1 position. ${ }^{21}$ All of these components are found in 1-palmitoyl-2-oleoyl-phosphocholine (POPC), which is why this lipid will be the representative lipid used in this study. All-atom molecular dynamics (MD) simulations will be used to describe how cholesterol affects the stability of a bilayer comprised of increasing amounts of 
Class B oxPLs. Atomistic MD simulations of lipid bilayer systems yield results that are comparable with experiments such as nuclear magnetic resonance (NMR), electron paramagnetic resonance and fluorescence measurements for parameters such as correlation time, deuterium order parameter, area per lipid (APL) and bilayer thickness. ${ }^{8}$ All-atom MD simulations of bilayers comprised of 30 mol\% cholesterol and 0, 20, 40, and 60 mol\% of oxPLs will be employed in this study to determine the role of cholesterol in the stabilization of a POPC lipid bilayer under an increasing load of Class B oxPLs.

\section{METHODS}

\subsection{Simulated systems}

In this work, bilayers comprised of 1-palmitoyl-2-oleoyl-phosphatidylcholine (POPC), 1palmitoyl-2-(9'-oxo-nonanoyl)-sn-glycero-3-phosphatidylcholine $\quad$ (PoxnoPC), 1-palmitoyl-2azelaoyl-sn-glycero-3-phosphatidylcholine (PazePC), and cholesterol were subjected to molecular dynamics simulations. The chemical structures of all molecules are shown in Figure 1. Each bilayer contained a total of 128 lipid molecules, with 30 mol\% of cholesterol. One system ( 0 mol\% oxPL) contained 90 POPC lipids, whereas the subsequent systems contained increasing amounts of the PoxnoPC and PazaPC in equal measure to represent lipid oxidation levels of 20, 40 and 60 mol\%. The 20 mol\% oxPL system contained 12 of each oxidized lipid, the 40 mol\% oxPL system contained 26 of each oxidized lipid, and the 60 mol\% oxPL system contained 38 of each oxidized lipid. A summary of the molecular composition of each system under investigation can be found in Table $\mathbf{1}$. Each system was solvated in water including $150 \mathrm{mM} \mathrm{NaCl}$ was added to mimic physiological salt concentration, which corresponded to a solvent comprised of $14 \mathrm{Na}^{+}, 14 \mathrm{Cl}^{-}$, and 5092 water molecules in a rectangular box.

Table 1. Molecular composition of the systems investigated in this study.

\section{System}

Number of Molecules

Box

Dimensions

(nm) 


\begin{tabular}{cccccccccc}
\hline & & & \multicolumn{2}{c}{ Oxidized Lipids } & \multicolumn{2}{c}{ Ions } & Water & \\
\cline { 5 - 8 } & & & PoxnoPC & PazaPC & $\mathrm{Na}^{+}$ & $\mathrm{Cl}^{-}$ & & \\
\hline 0 mol\% oxPL & 90 & 38 & - & - & 14 & 14 & 5092 & $6.52,6.52,7.24$ \\
\hline 20 mol\% oxPL & 66 & 38 & 12 & 12 & 14 & 14 & 5092 & $6.57,6.57,7.01$ \\
\hline 40 mol\% oxPL & 38 & 38 & 26 & 26 & 14 & 14 & 5092 & $6.24,6.24,7.59$ \\
60 mol\% oxPL & 14 & 38 & 38 & 38 & 14 & 14 & 5092 & $6.15,6.15,7.55$ \\
\hline
\end{tabular}

\subsection{Force field}

The parameters of all molecules used in this study are compatible with the OPLS-AA force field. ${ }^{22,23}$ The parameters for POPC, PoxnoPC, and PazaPC were derived as described previously, with the standard OPLS-AA parameters supplemented for the respective aldehyde and carboxylic acid groups in the case of PoxnoPC and PazaPC, respectively. The original OPLS-AA parameters for cholesterol were used, and the TIP3P model was used for water. ${ }^{24}$

\section{$\underline{2.3 \text { Simulation protocol }}$}

All MD simulations were carried out with version 4.6.x of the GROMACS program package and adhered to the same protocol, described as follows. ${ }^{25-27}$ A 500 ns MD simulation was run for the systems that contained 0 and 20 mol\% oxPLs with the intention of using the first $200 \mathrm{~ns}$ for equilibration. When it was observed that these systems were well-equilibrated within $100 \mathrm{ns,}$ as shown in Figure S1. it was decided to reduce the equilibration time of the systems containing 40 and $60 \mathrm{~mol} \%$ oxPLs to $100 \mathrm{~ns}$. As such, the final 300 ns of each trajectory was analyzed as described in section Analysis. During this, the LINCS algorithm ${ }^{28}$ was employed to constrain the length of all bonds during the simulation to enable a 2 fs time step to be used. To control pressure we employed the isobaric-isothermal ensemble under a constant pressure of 1 bar, and scaled the box volume semiisotropically using the Parrinello-Rahman barostat. ${ }^{29}$ The temperature of the simulation was set to $310 \mathrm{~K}$ and maintained as such by using the Nosé-Hoover thermostat, with the solvent and solute 
controlled independently. ${ }^{30,31}$ The list of non-bonded atom pairs were updated at every ten simulation steps, and the van der Waals interactions were cut-off at $1.0 \mathrm{~nm}$. Dispersion correction for both energy and pressure was used to reduce the dependencies on the cut-off length and to keep the model compatible with the OPLS-AA force-field. Periodic boundary conditions with the minimum image convention were used. The electrostatic interactions were evaluated using the particle-mesh Ewald summation, ${ }^{32}$ with an interpolation order of 6 , a direct sum tolerance of $10^{-5}$, and a cut-off of $1.0 \mathrm{~nm}$ was used for the real space calculations.

\section{$\underline{2.4 \text { Analysis }}$}

The stability of the area per lipid and membrane thickness over the entire simulation time was used to determine if each bilayer was equilibrated. The density of the lipid components and the assignment of the hydrogen bonds were calculated with the g_density and g_bond programs as implemented in GROMACS. A hydrogen bond was recorded when the angle between the hydrogen bonding donor, the hydrogen bonding hydrogen, and the hydrogen bonding acceptor was between $150^{\circ}$ and $180^{\circ}$, and the distance between the donor and acceptor atoms was less than $0.35 \mathrm{~nm}$. The interdigitation of the two membrane leaflets was characterized by computing the overlap parameter, ${ }^{33}$

$$
q_{o i}(z)=4 \frac{q_{i}(z) q_{o}(z)}{\left[q_{i}(z)+q_{o}(z)\right]^{2}}
$$

where $\mathrm{z}$ is the coordinate in the membrane normal direction, and $\mathrm{q}_{\mathrm{i}}(\mathrm{z})$ and $\mathrm{q}_{\mathrm{o}}(\mathrm{z})$ are the density values of atoms belonging to the respective inner and outer leaflets calculated from a trajectory centered at the middle of a bilayer center. Integrating the overlap parameter along the membrane normal (z) from zero to $d$ yields the length scale,

$$
\bullet=\int_{0}^{d} q_{o i}(z) d z
$$

which describes the degree to which the two leaflets are interdigitated.

The time-averaged membrane thickness and area per lipid were computed using the 
g_lomepro program. ${ }^{34}$ This program uses a modified version of the GridMAT-MD algorithm to map the reference atom of each lipid onto a grid. ${ }^{35}$ The area occupied by each lipid is estimated by summing the area of all the cells of the grid assigned to the lipid of interest, whereas the bilayer thickness is computed by computing the difference between grid elements in the top and bottom leaflets along the normal vector of the bilayer surface. The phosphate atom of the respective phospholipid and the oxygen atom of the cholesterol molecule were used as reference atoms. The deuterium order parameter $S_{C D}$, is a bilayer parameter that provides information about the order of the acyl chains, which can also be obtained accurately from NMR experiments. ${ }^{36} \mathrm{~S}_{\mathrm{CD}}$, is defined as:

$$
S_{C D}=\left\langle\frac{3}{2}\left(\cos ^{2} \theta_{i}\right)-\frac{1}{2}\right\rangle
$$

where $\theta_{\mathrm{i}}$ is the angle between a carbon-deuterium bond (the carbon-hydrogen bond in simulations) of the $i$-th carbon atom and the bilayer normal. The angled brackets indicate averaging over time of the relevant $\mathrm{C}-\mathrm{D}$ bond in the bilayer.

The tilt angle of cholesterol and the acyl chains, the number of contacts between water and lipid moieties, and the penetration of water was determined with the g_bundle, g_contact, and the g_count programs, as implemented in the GROMACS program package. The g_bundle program is used to measure the angle of bundles of axes with respect to the $z$-axis, and in this study the axis bundles were the vectors defined by the first and last $\mathrm{C}$ atoms of the sn- 1 and sn- 2 acyl chains of each phospholipid and the vector defined by the carbon nearest to the hydroxyl group and the C5 of the methyl heptane in cholesterol. The g_contact program was used to count the number of contacts between water and the phosphatidylcholine, the entire acyl chains, the $-\mathrm{CH}_{3},-\mathrm{CHO}$, and $\mathrm{COOH}$ of the acyl chain termini, and cholesterol. A contact was recorded when the distance between the atoms in the respective groups was less than or equal to $0.6 \mathrm{~nm}$. The counts were divided by the number of each respective lipid present to enable the data at different oxidation levels to be compared. The penetration of the bilayer by water was estimated by defining the bilayer core region, which was 
determined by first computing the average position of the phosphate atom in each lipid over the course of the simulation (with g_lomepro). The average $z$-axis position of these points defined the mid-plane of the bilayer (the membrane lying in the $x y$-plane, and the membrane core was defined of the region where $z$ was within $0.5 \mathrm{~nm}$ of the mid-plane (above and below the mid-plane). The g_count program then monitored the water molecules that entered this core region. 


\section{RESULTS}

\section{System densities, leaflet interdigitation, and hydrogen bonding between lipids.}

The plots of the area per lipid and bilayer thickness as a function of time (Figure S1) demonstrated that the systems were well-equilibrated at the start of and throughout the analysis period. The density of water, cholesterol, phosphatidylcholine (the phospholipid head group) and the lipid tails of POPC, PoxnoPC, and PazePC along the z-axis of each system are shown in Figure 2. The density of water overlaps with the density of each of the other lipid components in all four systems. The amplitude of the density maximum of each bilayer component varies as expected with the changes in lipid composition, and the area under the curve corresponding to the density of cholesterol was also constant, as expected. The density curves of the head groups of each leaflet remained distinct from each other, whereas those of the tails overlapped, indicating the possibility of the lipid tails of both leaflets occupying the same space. The lipids of the belonging to the respective inner and outer leaflets indeed become increasingly interdigitized as a function of the oxPL content of each bilayer, as shown in Figure S2. The value of the overlap parameter, $\mathrm{q}_{\mathrm{oi}}$, of each bilayer is as follows: 0 mol\% oxPL (0.37), 20 mol\% oxPL (0.54), 40 mol\% oxPL, (0.80), with 60 mol\% oxPL (0.94) showing the highest degree of interdigitation. The density curves of the POPC tails and cholesterol of each leaflet remain easily distinguishable at $20 \mathrm{~mol} \%$ oxPL or less, but the respective density curves nearly become flat as the oxPL content increases to $60 \mathrm{~mol} \%$ indicating the overlapping of the leaflets, as shown in Figure 2.

The average number of hydrogen bonds between lipids increased from 0.42 to 3.75 bonds per lipid as the oxidized lipid content increased from 0 to 60 mol\% for each lipid, as shown in Figure 3A. This is largely driven by the increased number of hydrogen bonds formed by PoxnoPC and PazaPC, since the hydrogen bonding of POPC and cholesterol remain relatively constant as the oxPL content increased. Though smaller in magnitude, it is noteworthy that the hydrogen bonding 

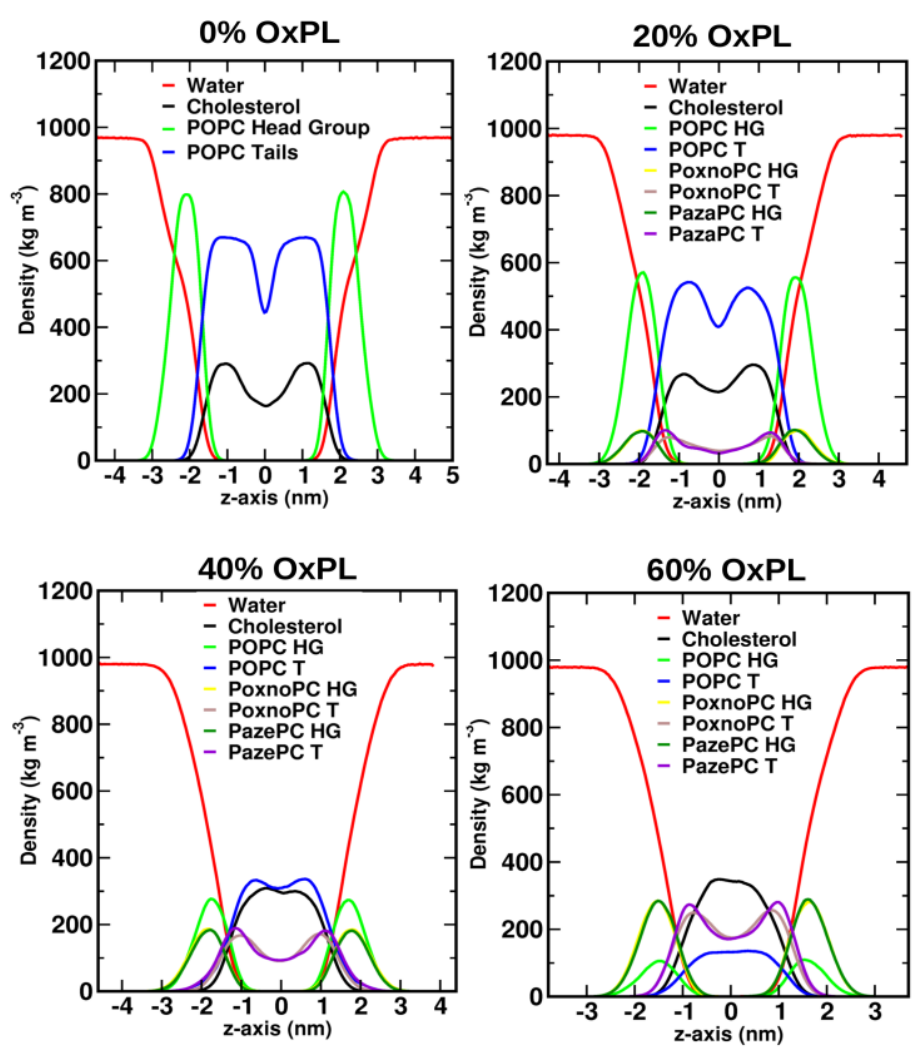

Figure 2. Mass density of each of the molecular components along the bilayer normal (along the z-axis) of each system. The phospholipids are further separated into their glycerophosphatidylcholine head groups and the acyl chains tail groups. The difference in amplitude between the leaflets of the cholesterol density at 20 mol\% oxPL can be attributed to a cholesterol flip from one leaflet to the other.

of cholesterol increased gradually from 0.30 to 0.35 hydrogen bonds with increasing oxPL, whereas the hydrogen bonding of POPC was significantly higher at 20 and 40 mol\% oxPL $(0.22$ and 0.20 hydrogen bonds, respectively) than that at 0 and $60 \mathrm{~mol} \%$ oxPL $(0.13$ and 0.07 hydrogen bonds, respectively).

The total number of hydrogen bonds between all pairwise lipids at any given time also increased with increasing oxidation. The number of hydrogen bonds between cholesterol and PoxnoPC and PazaPC increased, as did those between the PazaPC lipids, and those between PoxnoPC and PazaPC (Figure 3B). Only the hydrogen bonds between cholesterol and POPC decreased significantly. Interestingly, the hydrogen bonds between cholesterol molecules were at a 

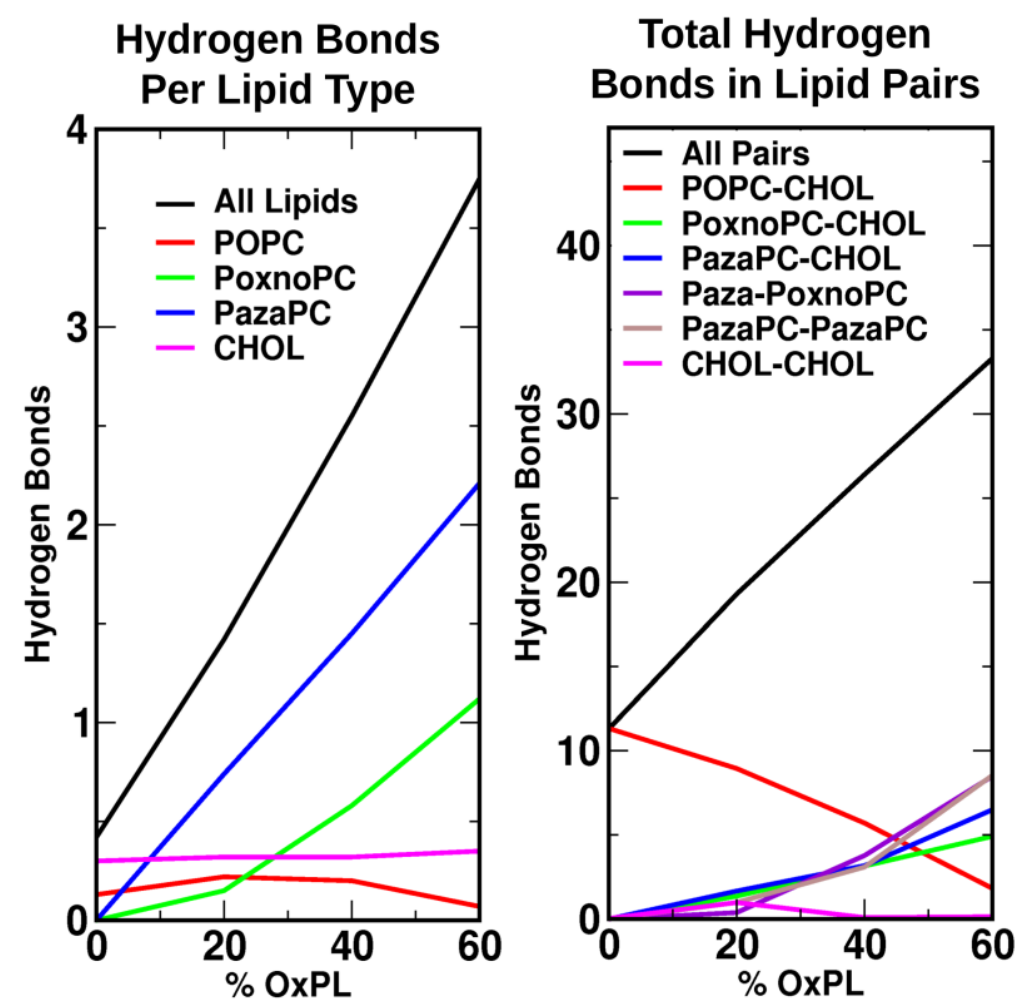

Figure 3. The average number of concurrent hydrogen bonds per each respective lipid molecule, and the number of concurrent hydrogen bonding pairs as a function of the percentage of OxPL content.

maximum of 0.98 hydrogen bonds at 20 mol\% oxPL, whereas it was below 0.15 hydrogen bonds at zero and higher oxidation levels.

\section{Bilayer thickness and area per lipid.}

The time-averaged thickness of the bilayer decreased as the oxPL content increased, and the range of the thickness of each bilayer determined during the simulation is shown in Figure 4. The median bilayer thickness decreased from $4.17 \mathrm{~nm}$ to $3.19 \mathrm{~nm}$ as the oxidation levels increased from 0 to $60 \mathrm{~mol} \%$ oxPL, whereas the mean area per lipid increased from $0.52 \mathrm{~nm}^{2}$ to $0.61 \mathrm{~nm}^{2}$ over the same oxidation range. Figure 4A shows that both the bilayer thickness and the area per lipid had a linear dependence on concentration from 0 to $40 \mathrm{~mol} \%$ oxPL, but oxidation had a diminished effect on the area per lipid at 60 mol\% oxPL than it did on bilayer thickness, which maintained a near linear relation to the oxPL content. Moreover, there was a significantly increased uncertainty in the area per lipid determination than in the uncertainty in the bilayer thickness. The range of the 


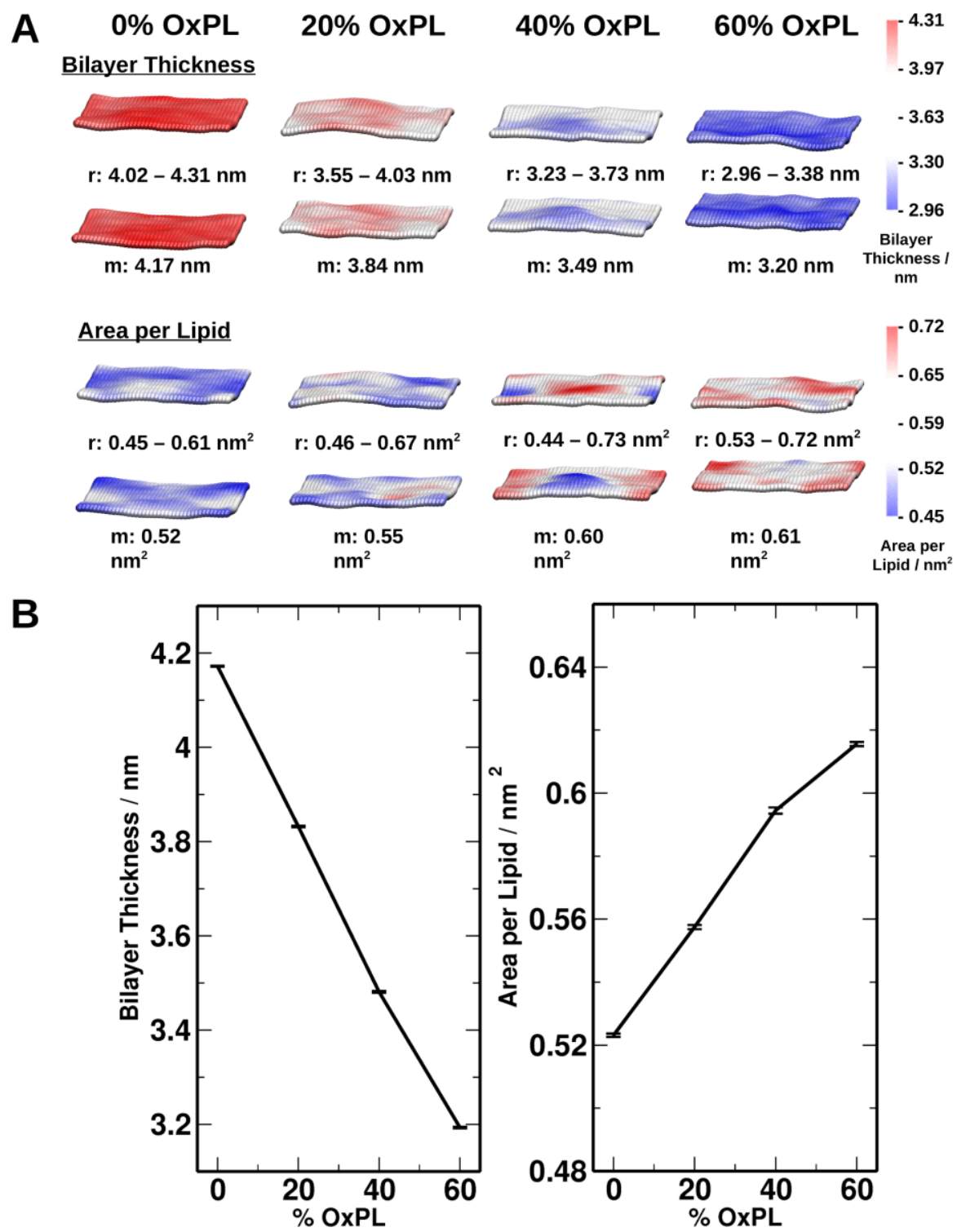

Figure 4. A)The time-averaged area per lipid and bilayer thickness of each system (top panel). The range of each respective value is color-coded, and the range (r) and median (m) values are also reported. B) The mean and standard error of the bilayer thickness and area per lipid as a function oxPL content (bottom panel).

thickness of each bilayer region indicated in Figure 4B shows that the thickness of the bilayer was the least uniform at $40 \mathrm{~mol} \%$ oxPL. This is also shown in the error bars in Figure 4A, which also reveals that the area per lipid was the least uniform at 40 mol\% oxPL. Figure $4 \mathbf{B}$ shows that regions with larger area per lipid values in one leaflet were directly above a complementary region of lower area per lipid values, and this effect was most prominent at 40 mol\% oxPL. 


\section{Deuterium order parameter and tilt angle of lipids tails and cholesterol.}

The sn- 1 acyl chain is not susceptible to oxidation and was present at identical concentrations in each bilayer. Maximum order was obtained at carbon seven of the sn- 1 acyl chain in POPC at 0 mol\% oxPL and at carbon five at 20 to $60 \mathrm{~mol} \%$ oxPL, which decreased from 0.332 to $0.189 \mathrm{~S}_{\mathrm{CD}}$ as the oxidation level increased (Figure 5). The $\mathrm{S}_{\mathrm{CD}}$ at 40 and $60 \mathrm{~mol} \%$ oxPL were nearly identical, whereas the order at $20 \mathrm{~mol} \%$ was near the midpoint of the maximum and minimum values, corresponding to 0 and 60 mol\% oxPL, respectively. The $\mathrm{S}_{\mathrm{CD}}$ of the sn- 1 chain in PoxnoPC and PazaPC followed similar patterns, including oxidation dependence, though the $\mathrm{S}_{\mathrm{CD}}$ of the sn-1 chain of PazaPC was lower at $20 \mathrm{~mol} \%$ and higher at $40 \mathrm{~mol} \%$ oxPL compared to the sn-1 chain of PoxnoPC. The $\mathrm{S}_{\mathrm{CD}}$ of the sn-2 acyl chain is distorted by the double bond in POPC, and by being truncated in PoxnoPC and PazaPC. The same tendency of the $\mathrm{S}_{\mathrm{CD}}$ was observed in the sn- 2 chain of POPC as was observed in the sn-1 chain of POPC (Figure 5). However, 20 mol\% oxPL had no effect on the sn-2 chain of PoxnoPC, whereas 40 and 60 mol\% oxPL caused a relatively large and nearidentical decrease in the order. The $\mathrm{S}_{\mathrm{CD}}$ of the sn-2 chain in PazaPC was much lower than that of POPC, however all levels of oxidation had a similar effect on its order.

The frequency of the tilt angle measurements for the sn- 1 and sn- 2 acyl chains, and cholesterol are shown in a histogram in Figure 6. At 0 mol\% oxPL, the most frequently measured tilt angle of the POPC acyl chains and cholesterol was near $18^{\circ}$, although smaller angles were more frequent for the longer sn- 1 chain than for the sn- 2 chain or for cholesterol, and the curve of the POPC sn-1 chain 

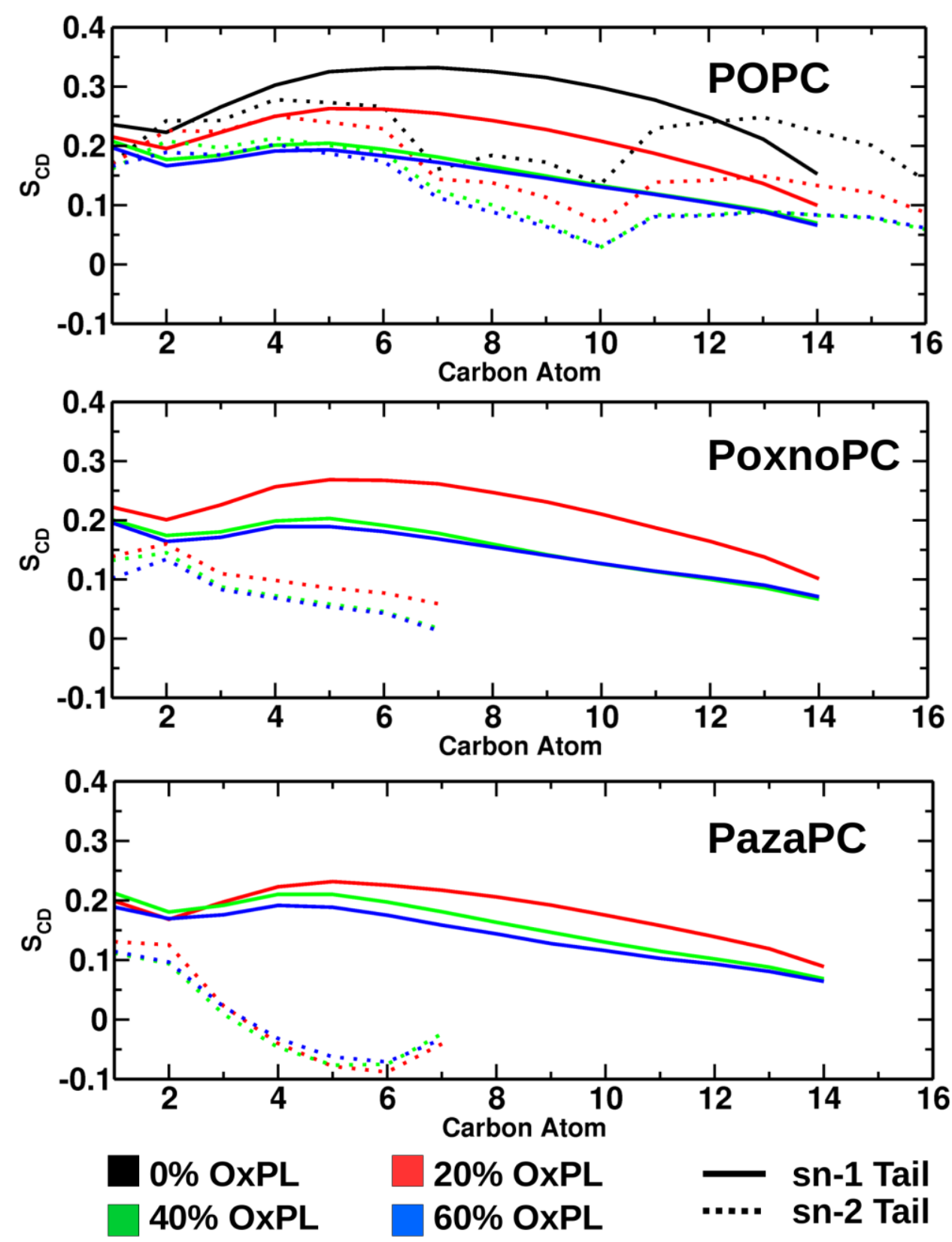

tailed-off at angles greater than $40^{\circ}$. The other POPC sn-1 peaks were right-shifted by approximately 10 degrees, though their amplitudes decreased with increasing oxidation. The right-shifting of the peaks was also observed for the POPC sn-2 tail, but significantly less so for cholesterol. The tailingoff of the peaks occurred at higher angles (further to the right) as the oxidation levels increased. However, at 60 mol\% oxPL the amplitude increases at angles greater

Figure 5. Deuterium order parameter of the lipid tails in each of the four color-coded membrane systems. For each of the POPC, PoxnoPC, and PazaPC lipids the sn-1 acyl chain is shown in a solid line and the sn-2 acyl chain is shown as a dotted line. 
than $40^{\circ}$, therefore the tailing at these curves are best described as smaller, distinct peaks. Angles greater than $140^{\circ}$ were observed for cholesterol, which was shown to be the result of the translocation of one cholesterol molecule across the bilayer. The curves of the sn-1 chain of PoxnoPC and PazaPC were similar to those of POPC, whereas the curves of the sn- 2 chain of PoxnoPC and PazaPC showed a broader distribution of angles. Compared to that of PoxnoPC, the 

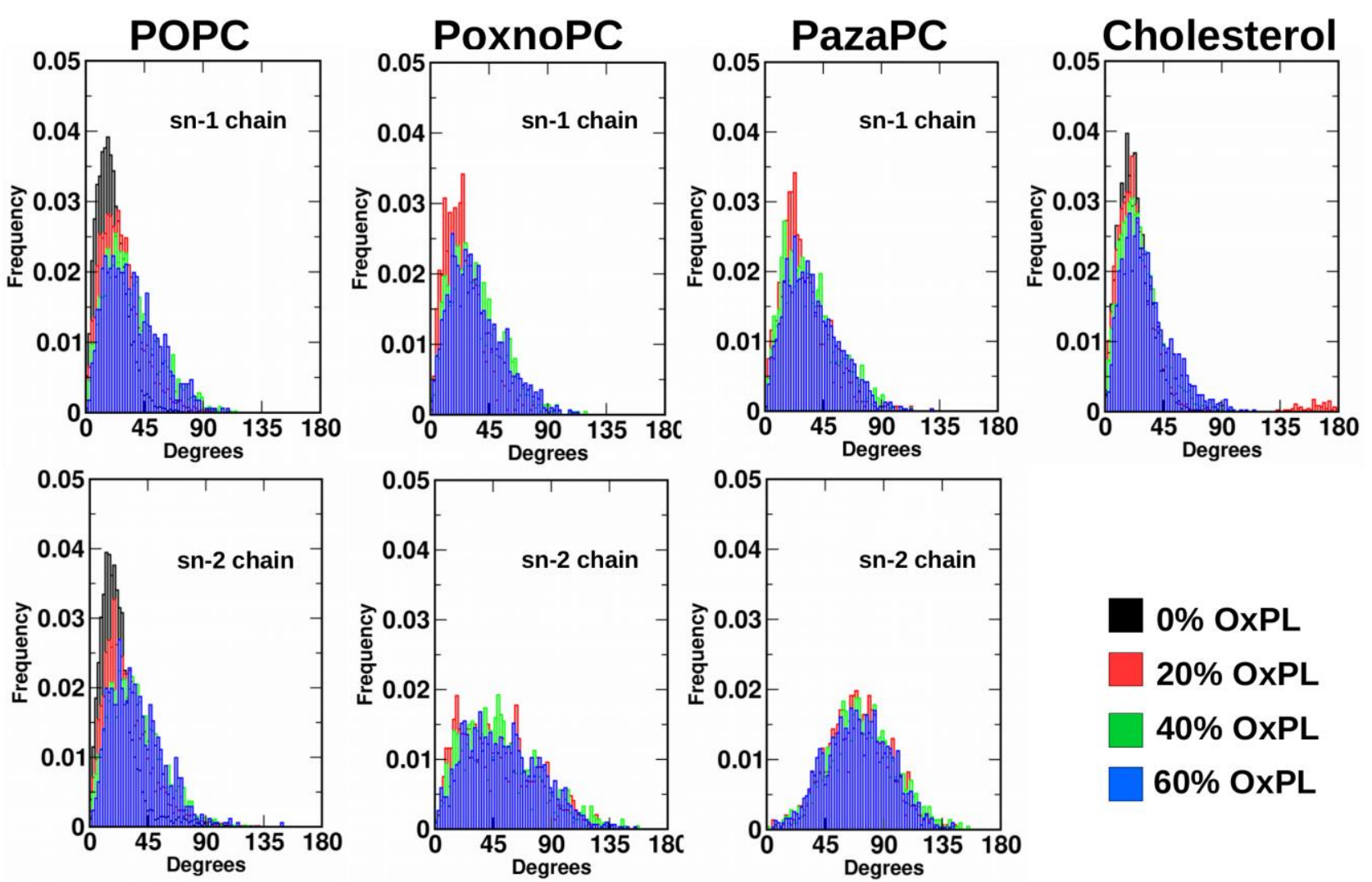

Figure 6. This distribution of the tilt angles of the sn-1 and sn-2 acyl chain of POPC and the tilt angle of cholesterol at each oxidation level.

curves of the sn-2 chain of PazaPC was more symmetrical with a peak amplitude near $80^{\circ}$, however the curves of PoxnoPC and PazaPC showed a lesser dependence on the oxPL concentration than those of POPC and cholesterol showed.

\section{Lipid contact with water and water penetration.}

The number of contacts per each phosphatidylcholine, acyl chain tail, and acyl chain terminus of each of POPC, PoxnoPC, and PazaPC with water, and the contacts between water and each cholesterol molecule are shown in Figure 7. The number of water molecules that made contact with the head group of POPC approximately doubled as the oxPL content increased from 0 to $60 \mathrm{~mol} \%$. Water made more contacts with PoxnoPC than with PazaPC when $20 \mathrm{~mol} \%$ of the tails were oxidized, however the contacts between water and these lipids were equal at 40 and 60 mol\% oxPL, with the number of contacts with the head groups decreasing as the oxidation increased. Fewer 

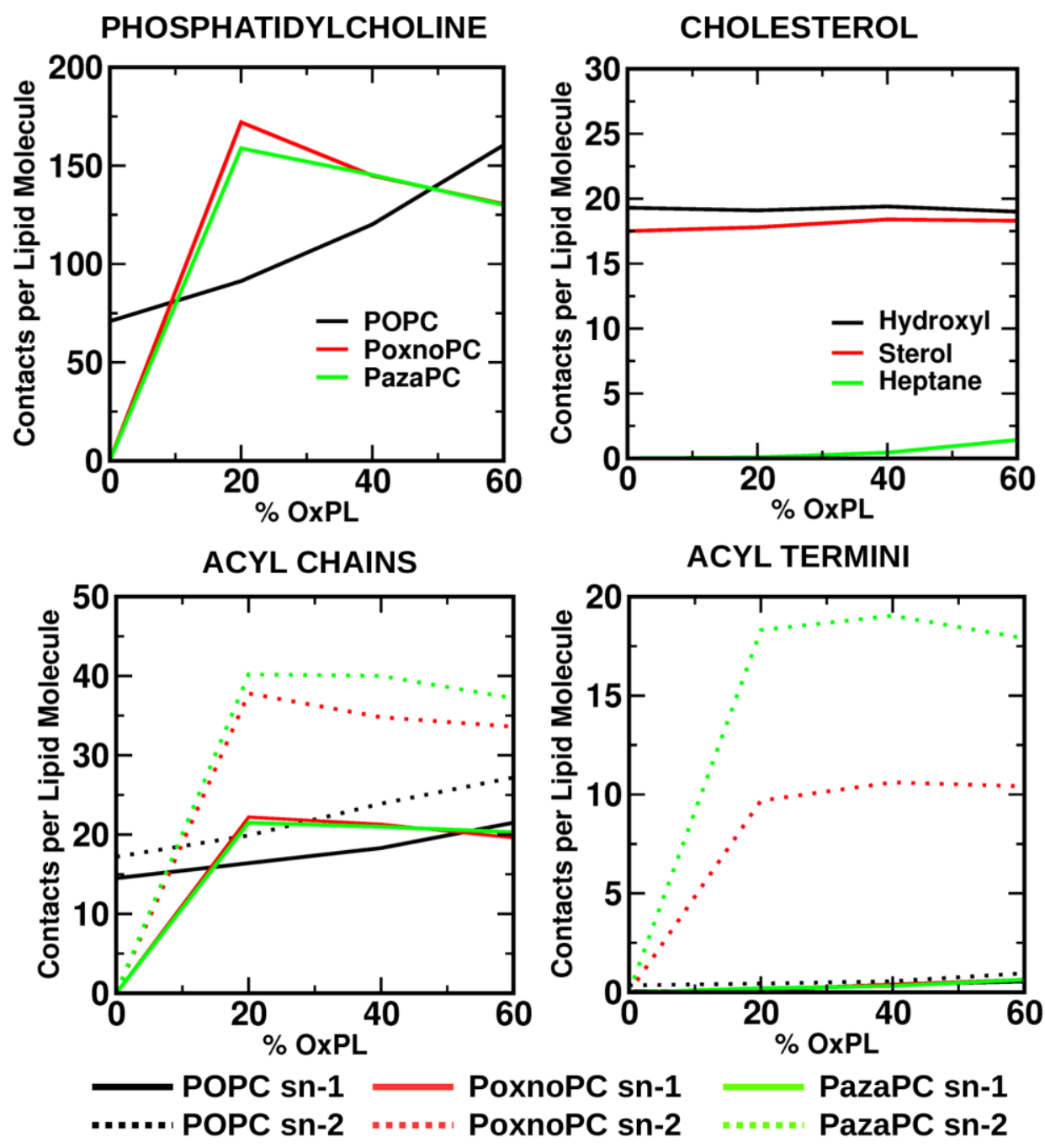

Figure 7. The number of concurrent contacts between water and the phosphatidylcholine, acyl chains and acyl termini of the POPC, PoxnoPC, and PazaPC phospholipids and between water and cholesterol as a function of the oxPL concentration. A contact was recorded when the distance between the atoms in the respective groups was less than or equal to $0.6 \mathrm{~nm}$.

contacts were made with the acyl chains, though the trends were similar (Figure 7). In POPC, 10$15 \%$ more contacts were made with the sn- 2 tail than with the sn- 1 tail, however this ratio was approximately $100 \%$ when the sn- 2 tails were oxidized, with more contacts made with the sn- 2 tail of PazaPC than with PoxnoPC. Twice as many contacts were made with the carboxylic acid of PazaPC than with the aldehyde of PoxnoPC, with negligible contact made between water and the methyl of the POPC sn-2 chain. Contacts between water and the hydroxyl, sterol, and 2methylheptane groups of cholesterol remained relatively constant as the oxPL content increased to 


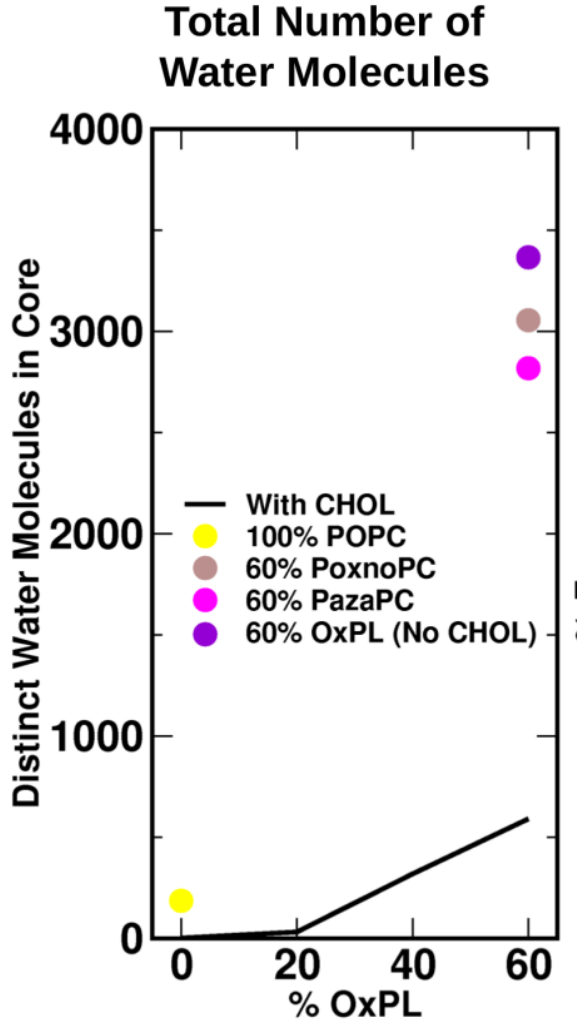

A

\section{Frequency of Frames With Each Amount of Water}

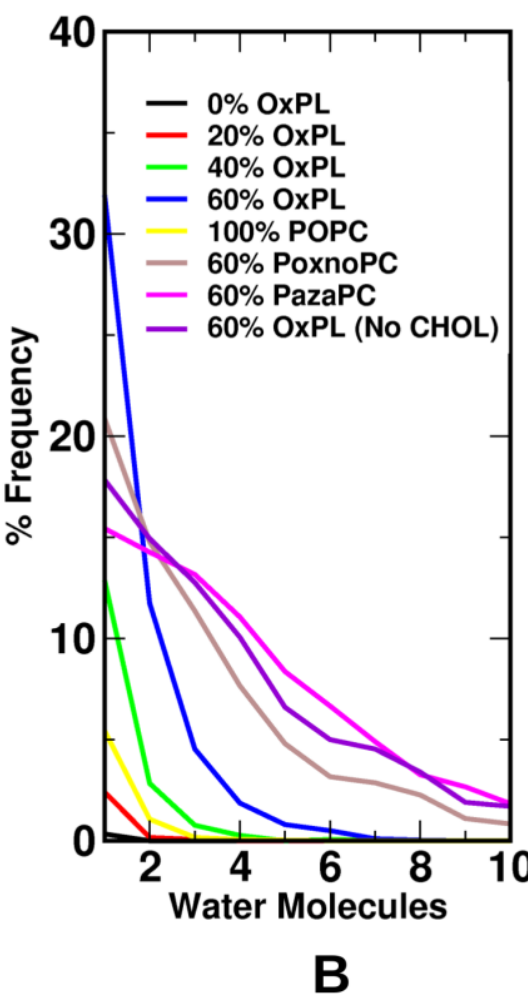

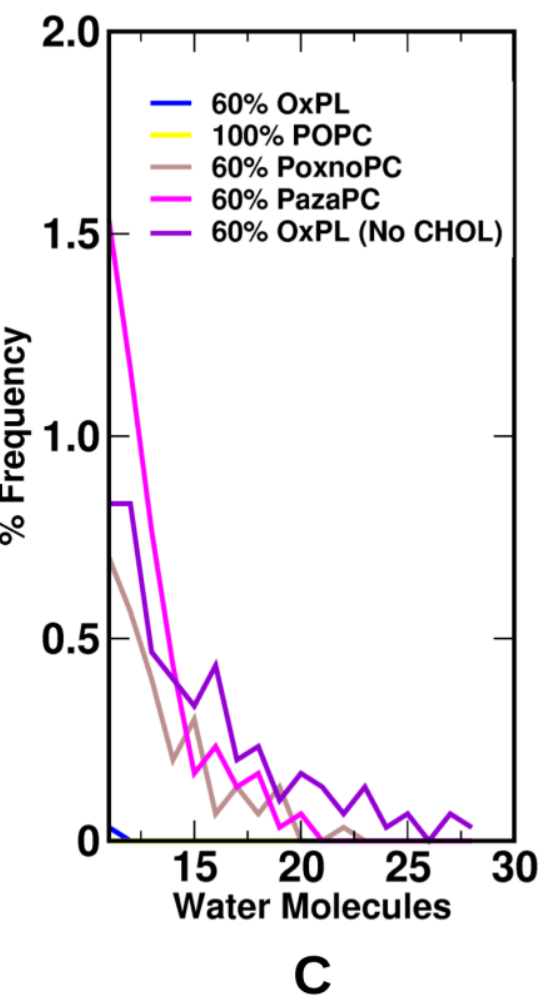

Figure 8. A) The total number of unique water molecules that enter the 1-nm-thick core region of the bilayer as a function of the oxPL concentration. B) and C) The frequency at which an increasing number of water molecules enters the core region of the bilayer at each level of oxidation. Note that panel C) is the extension of panel B) for the x-axis but with a smaller $\mathrm{y}$-axis scale. In panel C) the frequencies at 40 and $60 \mathrm{~mol} \%$ oxPL have already decayed to zero.

$40 \mathrm{~mol} \%$, though the number of contacts with hydroxyl and sterol decreased slightly, and those with heptane increased slightly at $60 \mathrm{~mol} \%$ oxPL.

The penetration of water into the bilayer core, a $1 \mathrm{~nm}$-thick region centered on the mid-plane of the bilayer, increased only marginally from 0 to $20 \mathrm{~mol} \%$ oxPL (Figure 8). However, the number of water molecules that enters this region increases from approximately 30 at $20 \mathrm{~mol} \%$ oxPL to approximately 600 at $60 \mathrm{~mol} \%$ over the course of the respective simulation. As a reference, only two water molecules entered this region at $0 \mathrm{~mol} \%$ oxPL. From the frames saved during the last $300 \mathrm{~ns}$ of each trajectory, there was at least one molecule of water in the core region in only $2.3 \%$ of them at $20 \mathrm{~mol} \%$ oxPL, but this increased to $13 \%$ of frames and $32 \%$ frames as the oxPL content increased to 
40 or $60 \mathrm{~mol} \%$. The number of frames decreased drastically for larger numbers of water molecules, as shown in Figure 8. Three water molecules were present in $0.76 \%$ of the frames at $40 \mathrm{~mol} \%$ oxPL, five water molecules were present at this frequency at $60 \mathrm{~mol} \%$ oxPL, whereas three water molecules were not observed at $20 \%$ oxPL or lower. The presence of six or more water molecules in the bilayers at $60 \mathrm{~mol} \%$ occurred in only $0.5 \%$ of simulation frames.

Far more water molecules were present in the bilayer core in the absence of cholesterol. Whereby only 2 distinct water molecules entered the core of the POPC bilayer with $30 \%$ cholesterol, 186 distinct water molecules entered this region in the POPC bilayer in absence of cholesterol. As shown in Figure 8, the introduction of $60 \%$ oxPL to the cholesterol-free bilayers increased the number of distinct water molecules in the bilayer core by more than 4.5 -fold compared to the number of water molecules in the core of the bilayers comprised of $60 \%$ oxPL and $30 \%$ cholesterol. The composition of the oxPL component as played a factor in the absence of cholesterol. More distinct water molecules were found in the bilayer core when the $60 \%$ oxPL was $50 \%$ PoxnoPC/PazaPC, followed by $60 \%$ oxPL comprised exclusively of PoxnoPC, and finally $60 \%$ oxPL comprised exclusively of PazaPC. Interestingly, at $60 \%$ oxPL, one molecule of water was found with the greatest frequency when cholesterol was present, and the occurrence of 2 water molecules in the core of the $60 \%$ oxPL bilayers was similar for the bilayer containing cholesterol and the three bilayers without cholesterol. However, the protective effect of cholesterol is demonstrated by its apparent ability to prevent three or more water molecules from entering the core, as the frequency of this occurrence decreases significantly, as shown in Figure 8B.

\section{Discussion and Conclusion}

Rather than represent the natural oxidation of a biological membrane, which would be comprised of a large array of lipid types, and even broader range of oxidized by-products, this study focuses on the effect of the conversion of the most common mammalian lipid, POPC into either 
PoxnoPC, or PazaPC, in well-defined model systems. The effect of this process on bilayer structure, lipid-water interactions and water penetration was studied as the percentage of PoxnoPC and PazaPC of the total lipid content was increased from 0 mol\% to 20,40 and $60 \mathrm{~mol} \%$ oxPL, with a fixed amount of $30 \mathrm{~mol} \%$ cholesterol present in each bilayer. The carboxylic acid remained protonated to determine the effect of this lipid on the bilayer structure before the proton is lost. This is reasonable since the protonated carboxylic acid lipid would have an increased stability in the hydrophobic environment of the inter-membrane space.

Increasing the total PoxnoPC and PazaPC content from 0 to 60 mol\% caused the bilayer thickness to decrease and area per lipid to increase. Even though these two parameters are related, the more uniform slope of the bilayer vs oxidation concentration compared to that of the area per lipid (APL) vs oxidation curve suggests that the bilayer thickness has a stronger correlation to the oxPL concentration than the APL does. As the oxPL concentration increases, the truncated sn-2 chains become more numerous and the hydrophobic region becomes thinner. This draws the polar head groups closer together, and thus makes the membrane thinner. The convergence of the density of the POPC tails and cholesterol from a doublet to a single peak as the oxPL concentration increases is further evidence of the interdigitation of the terminal methyl groups, which has been observed in previous experiments, as the methyl groups lie on either side of each other instead of above and below each other along the z-axis. ${ }^{37}$ The phosphatidylcholine head group of POPC, PoxnoPC and PazaPC are identical, thus they should have the same degree of water intercalation throughout the head group and thus a similar number of interactions with water. However, when corrected for the number of lipid molecules in each system, the number of contacts with water (Figure 7) suggests that the oxPL head groups remain more accessible to water at $20 \mathrm{~mol} \%$ oxPL, but as the concentration of oxPL increases the oxPLs become less accessible to water, which could also be due to the increased interdigitation of the sn-1 tails of the oxPLs compared to that of POPC. The increased interdigitation 
with oxPL content could subsequently cause the APL to increase and level off, and as the proportion of interdigitized oxPLs increases with respect to the total number of lipids. Thus we propose that the oxidatively mediated decrease in bilayer thickness causes the corresponding increase in APL.

The deuterium order parameter is often used to describe the order of the acyl chains, where the higher magnitude of the $\mathrm{S}_{\mathrm{CD}}$ signal, the higher the order of the acyl chains in the bilayer is. Increasing the oxPL content of the bilayers from 0 to 60 mol\% decreased the order of the sn- 1 tails of POPC, PoxnoPC and PazaPC, and the sn-1 chain of PazaPC was generally more strongly affected by oxidation than that of PoxnoPC. The effect of oxidation on the order of the sn-2 tails was larger, particularly that of PazaPC, which indicates that the sn-2 of PazaPC was more likely to deviate further from the normal. This deviation is likely due to the stronger interactions of the carboxylic acid tail of PazaPC compared to the aldehyde tail of PoxnoPC, presumably with the oxygen molecules of glycerol and phosphate and water molecules close to the water-lipid interface. The increasing magnitude of the $S_{C D}$ towards the terminus of the sn-2 chain of PazaPC shows that the terminus of this chain is relatively fixed, at least compared to that of PoxnoPC, and this occurs in the sn-2 chain of PazaPC but not in PoxnoPC. This is suggestive of interactions such as hydrogen bonds between carboxyl and glycerol or phosphate instead of with water, however the carboxyl group can still make contact with water molecules near the bilayer-solvent interface that are within the contact cutoff of $0.6 \mathrm{~nm}$ (as shown in Figure 7).

The increased solvent exposure of the phosphatidylcholine of POPC compared to that of the oxPLs suggested that the oxPLs recede from the lipid-water interface towards the bilayer core. Concurrently, the number of hydrogen bonds between cholesterol and POPC decreases, whereas the number of hydrogen bonds with the oxPLs increases with increasing oxPL concentration. When considering the hydrogen bonding ability of cholesterol, only its hydroxyl group is capable of forming hydrogen bonds, therefore the transfer of cholesterol's hydrogen bonds from POPC to the oxPLs 
occurs at the lipid-water interface. Since the phosphatidylcholine head groups of POPC and the oxPLs are identical, cholesterol must go deeper into the membrane as the oxPL concentration increases and the bilayer thickness decreases. This is shown clearly and quantitatively in Figure 2. The increasing number of hydrogen bonds between cholesterol and the oxPLs as the concentration of the oxPLs increases should strengthen the bilayer, even as the membrane becomes thinner.

Of POPC, PoxnoPC, PazaPC and cholesterol, only PazaPC (carboxyl hydrogen) and cholesterol (hydroxyl hydrogen) contain a proton donor. The increase in hydrogen bonds per lipid molecule of PazaPC and PoxnoPC is due to the tendency of the carboxyl group of PazaPC to form hydrogen bonds with the glycerol oxygen atoms of POPC, PoxnoPC and PazaPC. Therefore, the number of hydrogen bonds increases with increasing oxPL mol\% is because more of the PazaPC carboxyl groups are interacting with the glycerol oxygen atoms, though it is still not clear why more hydrogen bonds occur per PazaPC lipid. The increasing number of hydrogen bonds between all pairs and those only involving PazaPC can also be attributed to the the increase in the mol\% of PazaPC. However, the slight drop in hydrogen bonds between cholesterol lipids indicate their higher tendency of forming hydrogen bonds with either of the phospholipids with increasing oxPL mol\%, though this effect seems to be small.

Nevertheless, the bilayer becomes increasingly impregnated with water as the oxPL concentration increases, as shown in Figure 8. However, it is interesting to note that the number of contacts between water and the acyl chains of PoxnoPC and PazaPC does not increase but instead slightly decreases with increasing oxPL concentration, when the number of contacts are corrected to the per lipid values. This suggests that at concentrations of up to $60 \mathrm{~mol} \%$ oxPL, the number of water molecules within the bilayer core is not sufficient to cause the bilayer to form a large pore or even collapse. Moreover, close visual inspection of the trajectories did not show the formation of a pore in any system. Figure 8 also indicates that in some cases water penetration changes very little at the 
oxPL concentrations below $20 \mathrm{~mol} \%$, but similar to what was shown in the contacts between water and the acyl chains (Figure 7), the increase in water in the bilayer core occurs linearly as the oxPL concentration reaches 60 mol\%. Figure 8 further reveals that in some cases more than eight molecules enter the bilayer only in $0.005 \%$ of the simulation time, and only at an oxPL concentration of 40 mol\% or greater. The molecules that entered the bilayer core at oxPL concentrations greater than 40 mol\% still remained part of the bulk water (the pre-defined $1.0 \mathrm{~nm}$ core region had the same thickness for each system, and as the bilayer thickness decreased the bulk water had a shorter distance to travel before reaching the core region). Figure 9 shows a snapshot of each system when it contained the most water molecules in its respective core region. Even in the systems with 40 and 60 mol\% oxPL that contained 14 and 12 water molecules, respectively, not more than 2 or 3 of them had dissociated from the bulk water, indicating that water came closer to the membrane core 'in bulk' rather than as an increasing flux of individual water molecules. As the area per lipid increases, this is correlated with the tilting of the acyl chains, which prevents stray molecules from entering the bilayer core. The most frequently observed tilt angle of the POPC acyl chains at 60 mol\% oxPL remained near $30^{\circ}$, about $10^{\circ}$ larger than those at 0 mol\% oxPL, but since the angles above $60^{\circ}$ occurred highly infrequently, it is unlikely that these tails entered the aqueous layer, which is also indicative of the bilayer stability as the oxPL concentrations increases to $60 \mathrm{~mol} \%$. Finally, although the amplitude decreases slightly, the most frequently observed peak angle remains at $20^{\circ}$ at all oxidation levels, indicative of a stable bilayer even under an increasing oxPL 


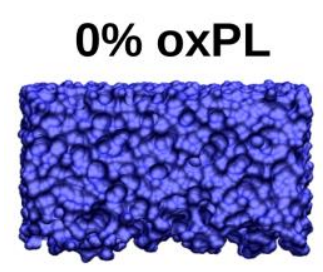

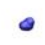

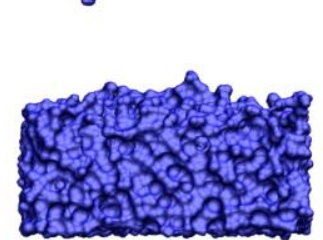

$20 \%$ oxPL
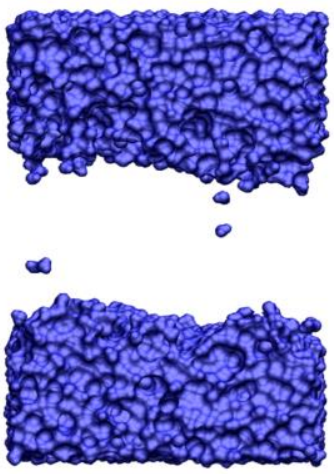

$40 \%$ oxPL

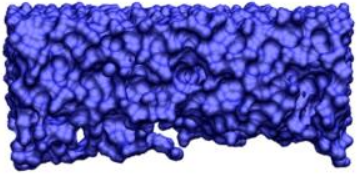

0

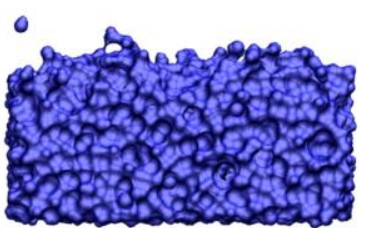

$60 \%$ oxPL

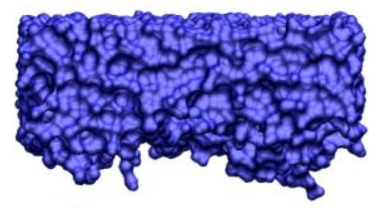

-

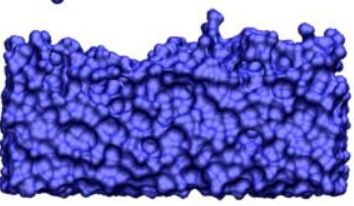

Figure 9. Snapshot of the water phase in each system with the most water molecules found in the respective core region.

load. The acyl chains would have a greater chance of accessing the solvent phase at angles greater than $90^{\circ}$, but an angle of greater than $120^{\circ}$ would be much more likely to access the solvent, as this angle would enable the acyl tip to go above (or below, in the lower leaflet) the glycerol groups to interact with the more solvent-accessible phosphate groups. Figure 6 shows only the sn- 2 chains of PazaPC, and to a lesser degree, PoxnoPC could accomplish this complete acyl chain reversal, but these instances were very infrequent, and surprising this maneuver showed little dependence on oxPL concentration. This means that the increasing the oxPL concentration did not increase the probability that a single truncated oxPL tail would invert, but instead lipid inversion events should remain proportional to the amount of oxPL present.

The changes to the hydrogen bonding and the deepening positioning of cholesterol into the in the bilayer as the oxPL content increases to $60 \%$ are strong supporting arguments towards the protective role of cholesterol against oxidative stress. However, these are confirmed by comparing the flux of water into the membrane of bilayers comprised of $60 \%$ oxPL and $30 \%$ cholesterol, to bilayers with $60 \%$ oxPL that are cholesterol-free. The result of this comparison is shown in Figure 8, wherein cholesterol-free bilayers comprised of $60 \%$ PoxnoPC, PazaPC, or a 1:1 ratio of PoxnoPC:PazaPC, all show a much higher rate of water of concurrent impregnation of 3 or more 
water molecules. Still, a channel did not form in any of the bilayers, which according to other studies, would require the oxidative truncation of both acyl chains, or an increase of oxPL concentration to $75 \%$ or higher, however, doubly-truncated lipids are rarely found in nature due to the saturation of the sn-1 acyl chain. ${ }^{17,38}$ It is possible that a channel may form under the conditions of this study had larger bilayers been used, however, it should be mentioned that bilayers in a study on Class B oxPLs that also used 128 lipids develop a channel at 75\% oxidation. This indicates that a 128-lipid bilayer (or even fewer) can reach the endpoint of pore formation under higher loads of oxPLs. ${ }^{17,38}$ An increased length in time-scale could also be requisite for pore formation, ${ }^{39}$ and combined MD/experimental studies have also demonstrated that oxPLs have a higher diffusion rates, ${ }^{13}$ which is slowed by cholesterol ${ }^{19}$ and could also influence the rate at which other compounds traverse the bilayer. ${ }^{40}$ Other observations such as chain reversal, ${ }^{15}$ and a decreased order parameter ${ }^{41}$ found in this study have also been demonstrated experimentally, however, the role of oxPLs in increasing APL and decreasing bilayer thickness has only been shown using MD simulations. ${ }^{8}$ Here we focused more on how cholesterol maintains the structure of intact bilayers, and the degree to which this is achieved is shown clearly over the $0 \%-60 \%$ oxidation range using the lipids in this study, and the supportive role of cholesterol has been shown experimentally. ${ }^{42}$ Oxidative levels of $60 \%$ may not occur in an entire cell, but it such levels are more likely to occur in a smaller region where a localized oxidative stress event could take place. Such a region could close to a source of free radicals such as mitochondria, or a location near the site of ROS production, as can be produced by the Fenton reaction. The aim of our results is to indicate what may happen in such an affected region of the membrane.

In conclusion, this study determined the structural changes that occur under oxidative stress in a membrane that contains cholesterol. This was done using model systems comprised of POPC, the most abundant mammalian diacylglycerol lipid and 30 mol\% cholesterol. The oxidative stress 
was increased by increasing the total PoxnoPC and PazaPC content in the lipids in fractions of 20, 40, and $60 \mathrm{~mol} \%$ of the total lipid content. In the presence of cholesterol, increasing the oxPLs decreased the acyl chain length, which caused the bilayer thickness to decrease with a strong dependence on the oxPL concentration. The corresponding increase in APL showed slightly weaker oxPL concentration dependence, and this increase can also be attributed to the increased interdigitation of the oxPL sn-1 tails, as well as the formation of hydrogen bonds and interactions between the truncated termini and the glycerol groups closer to the lipid water interface. The number of hydrogen bonds between cholesterol and the oxPLs also increase, with the lipid order parameter showing that PazaPC lipids showed a stronger tendency to do so than PoxnoPC lipids did. These hydrogen bonds stabilize the inter-lipid interactions that keep the bilayer intact even as its thickness and order decrease. Moreover, the tilt angle of cholesterol remains constant as the oxidative load increases. Water begins to enter the bilayer core as the oxPL concentration increases above 20 mol\%, and the number of water molecules that do this is linearly proportional to the oxPLs concentration, showing no sudden increase that would be evidence of pore formation or membrane disruption. Moreover, no pores were observed in the bilayer, demonstrating the stabilization effect of cholesterol against mechanical instability due to lipid oxidation. The inversion of the truncated PoxnoPC and PazaPC acyl chains is also linearly dependent on the oxPL content, since the distribution of the sn-2 tilt angles were only negligibly affected by the increasing oxPL concentration. This study provides more evidence on how cholesterol helps stabilize the mammalian plasma membrane under increasing oxidative load, preventing the occurrence of pore and micelle formation. The membrane protection demonstrated atomistically by the physiological levels of cholesterol used in this study can be indicative of how cholesterol protects multilaminar vesicles from micelle formation as shown experimentally. ${ }^{42}$ 


\section{ACKNOWLEDGEMENTS}

M. O. thanks the Helmholtz Postdoc Programmme, and the SoMoPro II programme, co-financed by the European Union and the South-Moravian Region, SoMoPro [Alzheimer's Lipids] - 6SA18009. This publication reflects only the author's views and the Union is not liable for any use that may be made of the information contained herein. W. K. and I.V. thank the Academy of Finland for financial support (the Finland Distinguished Professor (FiDiPro, Grant no. 263410) program, and the Center of Excellence (W.K., T.R, I.V.) funding (Grant no. 307415)). I.V. thanks the European Research Council (Advanced Grant CROWDED-PRO-LIPIDS (Grant no. 290974)). The CSC_-IT Centre for Science (Espoo, Finland) is acknowledged for excellent computational resources (project number tty3995). We also acknowledge grants of computer capacity from the Finnish Grid and Cloud Infrastructure (persistent identifier urn:nbn:fi:research-infras-201).

\section{$\underline{\text { REFERENCES }}$}

1. Downey, J. M. Free Radicals and their involvement during log-term myocardial ischemia and reperfusion. Annu. Rev. Physiol. 1990, 52, 487-504.

2. Uchida, K. 4-hydroxy-2-nonenal: a product and mediator of oxidative stress. Prog. Lipid Res. 42, 2003, 318-343.

3. Pham-Huy, L. A.; He, H.; Pham-Huy C. Free radicals, antioxidants in disease and health. Int. J. Biomed. Sci. 2008, 4, 89-96.

4. Deiger, H. -P.; Hermetter, A. Oxidized phospholipids: emerging lipid mediators in pathophysiology. Curr. Opin. Lipidol. 2008, 19, 289-294.

5. Kourie, J. I. Interaction of reactive oxygen species with ion transport mechanisms. Am. J. Physiol. 1998, 275, C1-C24.

6. Fruhwirth, G. O.; Loidl, A.; Hermetter, A. Oxidized phospholipids: from molecular properties to disease. Biochim. Biophys. Acta 2007, 1772, 718-736.

7. Grzelinska, I.; Bartosz, G.; Gwozdzinski, K.; Leyko, W. A spin-label study of the effect of gamma radiation on erythrocyte membrane. Int. J. Radiat. Biol. 1979, 36, 325-334.

8. Jurkiewicz, P.; Olzyńska, A.; Cwiklik, L.; Conte, E.; Jungwirth, P.; Megli, F. M.; Hof, M. Biophysics of lipid bilyers containing oxidatively modified phospholipids: insights from fluorescence and EPR experiments and from MD simulations. Biochim. Biophys. Acta 2012, 1818, 2388-2402.

9. Ikeda, M.; Kihara, A.; Igarashi, Y. Lipid asymetre of the eukaryotic plasma membrane: functions and related enzymes. Biol. Pharm. Bull. 2006, 8, 1542-1546.

10. Volinsky R.; Cwiklik, L.; Jurkiewicz, P.; Hof, M.; Jungwirth P.; Kinnunen, P. K. J. Oxidized phosphatidylcholines facilitate phospoholipid flip-flop in liposomes. Biophys. J. 2011, 101, 13761384. Megli, F. M.; Russo, L.; Conte, E. Spin labeling EPR studies of the properties of oxidized phospholipid-containing lipid vesicles. 2009, BBA Biomembranes 1788, 371-379. 
11. Megli, F. M.; Russo, L.; Conte, E. Spin labeling EPR studies of the properties of oxidized phospholipid-containing lipid vesicles. 2009, BBA Biomembranes 1788, 371-379.

12. Sabatini, K.; Juha-Pekka, M.; Megli, F. M.; Kinnunen, P. K. J. Characterization of two oxidatively modified phospholipids in mixed monolayers with DPPC. Biophys J. 2006, 90, 44884499.

13. Beranova, L.; Cwiklik, L.; Jurkiewicz, P.; Jungwirth, P.; Hof, M. Oxidation changes physical properties of phospholipid bilayers: fluorescence spectroscopy and molecular simulations. Langmuir 2010, 26, 6140-6144.

14. Khandelia, H.; Mouritsen, O. G. Lipid gymnastics: evidence of complete acyl chain reversal in oxidized phospholipids from molecular simulations, Biophys. J. 2009, 96, 2734-2743.

15. Mattila, J. P.; Sabatini, K.; Kinnunen, P. K. J. Interaction of cytochrome c with 1-palmitoyl-2azelaoyl-sn-glycero-3-phosphocholine: evidence for acyl chain reversal, Langmuir 2008, 24, 41574160.

16. Wong-Ekkabut, J.; Xu, Z. T.; Triampo, W.; Tang, I. M.; Tieleman, D. P.; Monticelli, L. Effect of lipid peroxidation on the properties of lipid bilayers: a molecular dynamics study, Biophys. J. 2007, 93, 4225-4236.

17. Cwiklik L. Jungwirth, P. Massive oxidation of phospholipid membranes leads to pore creation and bilayer disintigration. Chem. Phys Lett. 2010, 486, 99-103.

18. Khandelia, H.; Loubet, B.; Olzynska, A.; Jurkiewicz, P.; Hof, M. Paring of cholesterol with oxidized phospholipid species in lipid bilayers. Soft matter 2014, 10, 639-647.

19. Plochberger, B.; Stockner, T.; Chiantia, S.; Brameshuber, M.; Weghuber, J.; Hermetter, A.;

Schwille, P.; Schutz, G. J. Cholesterol slows down the lateral mobility of an oxidized phospholipid in a supported bilayer. Langmuir 2010, 26, 17322-17329.

20. Spector, A. A.; Yorek, M. A. Membrane lipid composition and cellular function. Lipid Res. 1985, 26, 1015-1035.

21. Gunstone, F. D.; Harwood, J. L.; Dijkstra, A. J. The lipid handbook with CD-ROM, $3^{\text {rd }}$ Edition Boca Raton, CRC Press 2007.

22. Jorgensen, W. L.; Maxwell, D. S.; Tirado-Rives, J. Development and testing of teh OPLS allatom force field on conformational energetics and properties of organic liquids. J. Am. Chem. Soc. 1996, 118, 11225-11236.

23. Kaminski, G. A.; Friesner, R. A.; Tirado-Rives, J.; Jorgensen, W. L. Evaluation and reparametrization of the OPLS-AA force field for proteins via comparison with accurate quantum chemical calculations on peptides. J. Phys. Chem., 2001, 105, 6474-6487.

24. Jorgensen, W. L.; Chandrasekhar, J.; Madura, J. D.; Impey, R. W.; Klein, M. L. Comparison of simple potential functions for simulating liquid water. J. Chem. Phys. 1983, 79, 926-935. 25. Van der Spoel, D.; Lindahl, E.; Hess, B; and the GRMACS development team, GROAMCS User Manual version 4.6.7, www.gromacs.org (2014).

26. Berendsen, H. J. C.; van der Spoel, D.; van Drunen, R. GROMACS: A message-passing parallel molecular dynamics implementation. Copm. Phys. Comm. 1995, 91, 43-56.

27. Hess, B.; Kutzner, C.; van der Spoel, D.; Lindahl, E. GROMACS 4: algorithms for highly efficient, load-balanced, and scalable molecular simulation. J. Chem. Theory Comput. 2008, 4, 435447.

28. Hess, B.; Kutzner, C.; van der Spoel, D.; Lindahl, E. GROMACS 4: algorithms for highly efficient, load-balanced, and scalable molecular simulation. J. Chem. Theory Comput. 2008, 4, 435447.

29. Parrinello, M.; Rahman, A. A polymorphic transitions in single crystals: a new molecular dynamics method. J. Appl. Phys. 1981, 52, 7182-7190. Nosé, S. A unified formulation of the constant temperature molecular dynamics methods. J. Chem. Phys. 1984, 81, 511-519. 
30. Nosé, S. A unified formulation of the constant temperature molecular dynamics methods. J. Chem. Phys. 1984, 81, 511-519.

31. Hoover, W. G. Canonical dynamics: equilibrium phase-space distributions. Phys. Rev. A 1985, 31, 1695-1697.

32. Essman, U.; Perera, L.; Berkowitz, M. L.; Darden, H. L. T.; Pedersen, L. G. A smooth particle mesh Ewald method. J. Phys. Chem. 1995, 103, 8577-8592.

33. Das, D.; Noroand, M. G.; Olmstead, P. D. Simulation Studies of Stratum Corneum Lipid Mixtures. Biophys. J. 2009, 97, 1941-1951.

34. Gapsys, V.; de Groot, B. L.; Briones, R. Computational analysis of local membrane properties. J. Comput. Aid. Mol. Des. 2013, 27, 845-858.

35. Allen, W.; Lemkul, J.; Bevan, D. GridMAT-MD: a grid-based membrane analysis tool for use with molecular dynamics. J. Comput. Chem. 2009, 30, 12; 1952-1958.

36. Davis, J. H. The description of membrane lipid conformation, order and dynamics by ${ }^{2} \mathrm{H}-\mathrm{NMR}$. Biochim. Biophys. Acta 1983, 737, 117-171.

37. Mason, P. R.; Walter, M. F.; Mason, P. E. Effect of oxidative stress on membrane stucture:

small angle x-ray diffraction analysis. Free Rad. Biol. Med. 1997, 23, 419-425.

38. Van der Paal, Neyts, E. C.; Verlackt, C. C. W.; Bogaerts, A. Effect of lipid peroxidation on membrane permeability of cancer and normal cells subjected to oxidative stress. Chem. Sci. 2016, 7, 489-498.

39. Boonnoy, P.; Jarerattanachat, V.; Karttunen, M.; Wong-ekkabut, J. Bilayer Deformation, Pores, and Micellation Induced by Oxidized Lipids. J. Phys Chem. Lett. 2015, 6, 4884-4888.

40. Gaede, H. C.; Gawrisch, K. Lateral Diffusion Rates of Lipid, Water, and a Hydrophobic Drug in a Multilamellar Liposome. Biophys. J. 2003, 85, 1734-1740.

41. Wratten, M.; van Ginkel, G.; van't Veld, A. A.; Bekker, A., van Faasen, E. E.; Sevanian, A.

Structural and dynamic effects of oxidatively modified phospholipids in unsaturated lipid membranes. Biochemistry 1992, 31, 10901-10907.

42. Megli, F. M.; Conte, E. Ishikawa, T. Cholesterol attenuates and prevents bilayer damage and breakdown in lipoperoxidized model membranes. A spin labeling EPR study. Biochim. Biophys. Acta 2011, 1808, 2267-2274. 